\title{
BMJ Open Association between potentially inappropriate medications at discharge and unplanned readmissions among hospitalised elderly patients at a single centre in Japan: a prospective observational study
}

\author{
Junpei Komagamine (D) , Taku Yabuki, Masaki Kobayashi
}

To cite: Komagamine J, Yabuki T, Kobayashi M. Association between potentially inappropriate medications at discharge and unplanned readmissions among hospitalised elderly patients at a single centre in Japan: a prospective observational study. BMJ Open 2019;9:e032574. doi:10.1136/ bmjopen-2019-032574

- Prepublication history and additional material for this paper are available online. To view these files, please visit the journal online (http://dx.doi. org/10.1136/bmjopen-2019032574).

Received 25 June 2019 Revised 24 October 2019 Accepted 24 October 2019

Check for updates

(C) Author(s) (or their employer(s)) 2019. Re-use permitted under CC BY-NC. No commercial re-use. See rights and permissions. Published by BMJ.

Internal Medicine, National Hospital Organization Tochigi Medical Center, Utsunomiya, Japan

Correspondence to Dr Junpei Komagamine; junpei0919@yahoo.co.jp

\section{ABSTRACT}

Objective To determine the prevalence of potentially inappropriate medication (PIM) use at admission and discharge among hospitalised elderly patients and evaluate the association between PIMs at discharge and unplanned readmission in Japan.

Design A prospective observational study conducted by using electronic medical records.

Participants All consecutive patients aged 65 years or older who were admitted to the internal medicine ward were included. Patients who were electively admitted for diagnostic procedures were excluded.

Main outcome measures The primary outcome was 30-day unplanned readmissions. The secondary outcome was the prevalence of any PIM use at admission and discharge. PIMs were defined based on the Beers Criteria. The association between any PIM use at discharge and the primary outcome was evaluated by using logistic regression.

Results Seven hundred thirty-nine eligible patients were included in this study. The median patient age was 82 years (IQR 74-88); 389 (52.6\%) were women, and the median Charlson Comorbidity Index was 2 (IQR 0-3). The proportions of patients taking any PIMs at admission and discharge were $47.2 \%$ and $32.2 \%$, respectively. Of all the patients, 39 (5.3\%) were readmitted within 30 days after discharge for the index hospitalisation. The use of PIMs at discharge was not associated with an increased risk of 30 -day readmission (OR $0.93 ; 95 \% \mathrm{Cl} 0.46$ to 1.87 ). This result did not change after adjusting for patient age, sex, number of medications, duration of hospital stay and comorbidities (OR 0.78; $95 \% \mathrm{Cl} 0.36$ to 1.66).

Conclusion The prevalence of any PIM use at discharge was high among hospitalised elderly patients in a Japanese hospital. Although the use of PIMs at discharge was not associated with an increased risk of unplanned readmission, given a lack of power of this study due to a low event rate, further studies investigating this association are needed.

Trial registration number UMIN000027189.
Strengths and limitations of this study

- This is the first prospective observational study investigating the use of potentially inappropriate medications and unplanned readmissions in Japan.

- When possible, we contacted the principal physicians prescribing the medications to collect accurate information on the medications.

- The $95 \% \mathrm{Cl}$ of the OR for the primary outcome was wide due to an unexpectedly low event rate.

- A single-centre study design limited the generalisability of the results.

- The data on readmission might be inaccurate due to the use of electronic medical records to acquire the data.

\section{INTRODUCTION}

Inappropriate prescribing, which encompasses potentially inappropriate medications (PIMs) and potentially inappropriate omissions, ${ }^{1}$ is a relevant public health concern for older patients. Potentially inappropriate medications are defined as medications that potentially have more harmful effects than beneficial effects for elderly individuals, who are defined as 65 years and older. ${ }^{2}$ The use of PIMs in elderly patients has been associated with an increased risk of several adverse outcomes. $^{3-6}$ Therefore, the use of PIMs should be avoided if possible. ${ }^{12}$

Nonetheless, the use of PIMs is common among elderly patients, ${ }^{78}$ particularly in longterm care facilities ${ }^{9}$ and acute care settings. ${ }^{10}$ The prevalence of PIMs has been reported to be $20 \%-70 \%$ in elderly patients with acute illnesses. ${ }^{10-15}$ Furthermore, the use of PIMs at discharge is also common because hospitalisation often contributes to an increased use of PIMs. ${ }^{16}$ However, few studies have ever been 
conducted to investigate the effect of PIM use at discharge from hospitalisation on patient outcomes. ${ }^{17-19}$ After hospital discharge, medication-related harm is common among elderly patients. ${ }^{20}{ }^{21}$ Furthermore, given that the use of PIMs is an independent risk factor for hospitalisation among elderly patients dwelling in the community and nursing care facilities, ${ }^{46}$ the use of PIMs at hospital discharge may increase the risk of unplanned readmissions. Therefore, we investigated the association between the use of PIMs at hospital discharge and unplanned readmissions among hospitalised elderly patients. In addition, we determined the prevalence of PIMs at admission and discharge among hospitalised elderly patients because there are few studies investigating these prevalence rates in Japan. ${ }^{1112}$

\section{METHODS}

\section{Study design and settings}

A prospective observational study was conducted by using the database of the National Hospital Organization Tochigi Medical Center from May 2017 to November 2018. The National Hospital Organization Tochigi Medical Center is a 350-bed community general hospital that is one of the largest acute care hospitals in Utsunomiya in Japan. This research was registered at the University Hospital Medical Information Network (UMIN) clinical registry on 29 April 2017.

\section{Inclusion and exclusion criteria}

All consecutive patients aged 65 years and older who were admitted to the internal medicine ward in our hospital from 1 May 2017 to 31 May 2018, and alive at discharge were included. For patients who experienced multiple admissions during the study period, only the first admission was included because the inclusion of multiple admissions for the same patient might result in excessive intensification of the effects of patient characteristics on the outcomes. Patients who were transferred to other hospitals or other wards in our hospital for more intensive or specialised care were excluded. In our hospital, patients were transferred from the internal medicine ward to other wards for surgical procedures. Because surgical patients might have a different risk of readmission than internal medicine patients, patients who were transferred to other wards in our hospital were excluded. We also excluded patients who were electively admitted for diagnostic or intervention procedures because the readmission risk for these patients is lower than that of patients hospitalised due to acute medical problems. Patients whose data regarding medications at admission or discharge were missing were also excluded. During the study period, 1707 adult hospitalised patients were screened. Of those, 739 patients were included in the final analysis (detailed information is shown in a online supplementary figure).

\section{Data collection}

We collected data from the electronic medical records of the National Hospital Organization Tochigi Medical
Center. Information on age, sex, Charlson Comorbidity Index (CCI) ${ }^{22}$ primary diagnosis for admission, social history and medical history were extracted from the electronic medical records at the time of the index admission. For information on a disease or syndrome, a patient was considered not to have a disease or syndrome if there was no documentation of them in the electronic medical records. In our hospital, pharmacists routinely compile a comprehensive medication list after admission. All medications used during hospitalisation were managed by the principal physician caring for the patients. Therefore, information on medication at admission was obtained by using the comprehensive medication list or medical records documented by the physicians, while information on medication at discharge was based on the discharge prescription issued by the principal physicians. If information on prescribed medications was missing or unclear in the electronic medical records, we contacted the principal physicians and collected accurate information on the medications if possible. We included regularly used medications but not as-needed medications because collecting accurate information on the frequency of use of as-needed medications was not possible. Topical medications, eye drops and intranasal infusers were excluded. Over-the-counter (OTC) drugs were also excluded because information on these drugs was not sufficiently collected as part of the usual care in this hospital. Information on 30-day readmission was also collected using the electronic medical records of the National Hospital Organization Tochigi Medical Center until 30 November 2018.

\section{Outcome measures}

The primary outcome was 30-day unplanned readmission. Unplanned readmissions to the emergency department that did not result in hospitalisation were not included. In the original version of the protocol, we planned to evaluate the 30-day unplanned readmissions only. However, the 30-day unplanned readmission rate was unexpectedly low during the study period. Therefore, 90-day readmissions were also evaluated. The secondary outcome was any use of PIMs. The prevalence of any PIM use at admission and discharge was evaluated. Based on the previous research, ${ }^{11}$ we defined PIMs based on the 2015 Beers Criteria of the American Geriatric Society. ${ }^{2}$ Based on a previous study, ${ }^{11}$ we used only two of the five parts of the Beers Criteria, that is, PIM use in older adults and PIM use in older adults due to drug-disease or drugsyndrome interactions that may exacerbate the disease or syndrome. However, regarding proton-pump inhibitors (PPIs), based on previous studies ${ }^{1123}$ and guidelines, ${ }^{24-26}$ their use was judged to be potentially inappropriate if there were none of the following indications: (1) active peptic ulcer disease; (2) gastro-oesophageal reflux disease; (3) use of non-steroidal anti-inflammatory drugs; (4) use of antiplatelet therapy and at least one risk factor (history of peptic ulcer disease, dual antiplatelet therapy or concomitant use of anticoagulants or corticosteroids); 
(5) pathological hypersecretory conditions and (6) Helicobacter pylori eradication therapy.

\section{Statistical analysis}

We estimated that a sample of 650 patients would provide the study with a power of at least $80 \%$ to show an absolute difference of $8 \%$ for the primary outcome between the patients who took any PIMs at discharge and patients who did not. This estimate was determined assuming that $13 \%$ of elderly patients who took any PIMs at discharge would be readmitted within 30 days after discharge of the index hospitalisation (based on unpublished data of previous research ${ }^{11}$ ) and that $15 \%$ of hospitalised elderly patients with a medical illness would die during their hospitalisation.

The baseline and demographic characteristics of the study population were summarised by standard descriptive summaries (eg, the median and IQR for continuous variables and percentages for categorical variables). For the primary outcome, the proportion of patients who had unplanned readmission within 30 days after the index hospital discharge was calculated based on the presence of any PIM use at discharge. For the secondary outcome, the proportion of patients taking any PIMs at admission and discharge was calculated. The 95\% CIs were calculated for these outcomes. The rates of reduction in the prevalence of any PIM use from admission to discharge were calculated, and a comparison between admission and discharge regarding the prevalence of PIM use was performed using the exact McNemar test. The number of medications at admission was compared with the number of medications at discharge using the Wilcoxon rank-sum test. These analyses for the secondary outcome were also conducted for subgroups of patients classified based on their principal diagnosis for admission. For the primary outcome, the proportion of patients who had unplanned readmission within 30 days after discharge was compared using Fisher's exact test between patients who took any PIMs at discharge and patients who did not. Multivariate analysis using binary logistic regression was also conducted to examine the association between the primary outcome and any PIM use at discharge by adjusting for age, sex, CCI, polypharmacy at discharge and duration of hospitalisation. Polypharmacy was defined as five or more medications based on a previous study. ${ }^{27}$ The same analysis was also performed for the 90-day unplanned readmissions.

For the definition of PIM regarding PPIs, we used the original criteria instead of the 2015 Beers Criteria. Therefore, the association between unplanned readmissions and the use of PIMs based only on the 2015 Beers Criteria without conditional criteria regarding PPI use was also investigated. These analyses were performed using Stata V.15 (LightStone, Tokyo, Japan) or Excel statistical software package V.2.11 (Bellcurve for Excel; Social Survey Research Information, Tokyo, Japan). The threshold for significance was set at $5 \%$.

\section{Patient and public involvement statement}

No patients were involved in determining the research question or outcome measures, nor were they involved in developing plans to design or implement the study. No patients were asked for advice during the interpretation or writing up of the results of this study. We have no plans to disseminate the results of this research to study participants or the relevant patient community.

\section{RESULTS}

The baseline characteristics of the 739 elderly hospitalised patients are shown in table 1 . Of those, the median patient age was 82 years (IQR 74-88), 389 (52.6\%) were women, $93(12.6 \%)$ were institutional residents, the median CCI was 2 (IQR 0-3) and the median number of medications at admission was 5 (IQR 3-8). The most common reasons for admission were acute heart failure $(n=95,12.9 \%)$, pneumonia or pneumonitis $(n=64,8.7 \%)$, stroke $(n=53$, $7.2 \%)$, acute coronary syndrome $(n=41,5.6 \%)$ or urinary tract infection $(\mathrm{n}=39,5.3 \%)$.

The median number of medications at admission and discharge was 5 (IQR 3-8) and 4 (IQR 2-6), respectively. The number of medications significantly decreased from admission to discharge (see the online supplementary table S1). Table 2 shows the prevalence of any PIM use at admission and discharge. The proportions of patients taking any PIMs at admission and discharge were $47.3 \%$ and $32.2 \%$, respectively. The prevalence of any PIM use was significantly reduced from admission to discharge (reduction rate 0.32 ; $95 \%$ CI 0.25 to 0.38 ) (detailed information is shown in a online supplementary table S2). The most common subcategories of PIMs at discharge were PPIs $(n=140,18.9 \%)$, benzodiazepines $(n=64,8.7 \%)$, non-benzodiazepine hypnotics $(\mathrm{n}=29,3.9 \%)$ and antipsychotics $(n=15,2.0 \%)$.

Of all patients, unplanned readmission within 30 days and 90 days after the index hospital discharge occurred in $39(5.3 \%)$ and $98(13.3 \%)$ patients, respectively (see the online supplementary table S3). The 30-day unplanned readmission rate in patients who took any PIMs at discharge and those who did not take any PIMs were $5.0 \%$ and $5.4 \%$, respectively. Table 3 shows the results of univariate and multivariate analyses for predictive factors for the 30-day and 90-day unplanned readmissions. Any PIM use at discharge was not significantly associated with an increased risk of 30-day unplanned readmissions (OR 0.93 ; $95 \%$ CI 0.46 to 1.87 ) and 90-day unplanned readmissions (OR $0.78 ; 95 \%$ CI 0.48 to 1.24 ). These results did not change after adjusting for patient age, sex, duration of hospitalisation, polypharmacy and comorbidity. When the original definition of PIM regarding PPI use was not used, any PIM use at discharge was not significantly associated with an increased risk of 30-day unplanned readmissions (OR 0.78; 95\% CI 0.29 to 2.15 ) and 90-day unplanned readmissions (OR 0.52; 95\% CI 0.26 to 1.07 ) (see the online supplementary table $\mathrm{S} 4$ ). 
Table 1 Characteristics of the 739 elderly patients admitted to the internal medicine ward based on the occurrence of a 30day unplanned readmission after the index hospital discharge

\begin{tabular}{|c|c|c|c|}
\hline \multirow[b]{2}{*}{ Characteristics } & \multirow{2}{*}{$\begin{array}{l}\text { Total } \\
(\mathrm{n}=739)\end{array}$} & \multicolumn{2}{|c|}{ The occurrence of a 30-day unplanned readmission } \\
\hline & & Yes $(n=39)$ & No $(n=700)$ \\
\hline Age (year), median (IQR) & $82(74-88)$ & $83(75-89)$ & $82(74-88)$ \\
\hline Women, n (\%) & $389(52.6)$ & $13(33.3)$ & $376(53.7)$ \\
\hline Institutional resident, $\mathrm{n}(\%)$ & $93(12.6)$ & $7(17.9)$ & $86(12.3)$ \\
\hline Charlson Comorbidity Index, median (IQR) & $2(0-3)$ & $3(1-4)$ & $1(0-3)$ \\
\hline \multicolumn{4}{|l|}{ Number of medications at admission } \\
\hline Median (IQR) & $5(3-8)$ & $6(3-9)$ & $5(3-8)$ \\
\hline Five or more medications, $n(\%)$ & $446(60.4)$ & $25(64.1)$ & $421(60.1)$ \\
\hline \multicolumn{4}{|l|}{ Number of medications at discharge } \\
\hline Median (IQR) & $4(2-6)$ & $4(1-7)$ & $4(2-6)$ \\
\hline Five or more medications, $\mathrm{n}(\%)$ & $288(39.0)$ & $19(48.7)$ & $269(38.4)$ \\
\hline Current smoker, $\mathrm{n}(\%)$ & $78(10.6)$ & $5(12.8)$ & $73(10.4)$ \\
\hline Regular alcohol drinker*, n (\%) & $116(15.7)$ & $8(20.5)$ & $108(15.5)$ \\
\hline \multicolumn{4}{|l|}{ Principal diagnosis for admissiont, $\mathrm{n}(\%)$} \\
\hline Acute heart failure & $95(12.9)$ & $7(17.9)$ & $88(12.6)$ \\
\hline Pneumonia or pneumonitis & $64(8.7)$ & $3(7.7)$ & $61(8.7)$ \\
\hline Stroke $\mathrm{e}^{\ddagger}$ & $53(7.2)$ & $2(5.1)$ & $51(7.3)$ \\
\hline Acute coronary syndrome & $41(5.6)$ & $3(7.7)$ & $38(5.4)$ \\
\hline Urinary tract infection & $39(5.3)$ & $1(2.6)$ & $38(5.4)$ \\
\hline \multicolumn{4}{|l|}{ Medical history, n (\%) } \\
\hline Stroke $\ddagger$ & $134(18.1)$ & 7 (17.9) & $127(18.1)$ \\
\hline Dementia & $159(21.5)$ & $13(33.3)$ & $146(20.9)$ \\
\hline Diabetes mellitus & $200(27.1)$ & $13(33.3)$ & $187(26.7)$ \\
\hline Ischaemic heart disease§ & $54(7.3)$ & $3(7.7)$ & $51(7.3)$ \\
\hline Asthma or COPD & $93(12.6)$ & $5(12.8)$ & $88(12.6)$ \\
\hline Heart failure & $99(13.4)$ & $8(20.5)$ & $91(13.0)$ \\
\hline Atrial fibrillation & $76(10.3)$ & $3(7.7)$ & $73(10.4)$ \\
\hline Hypertension & $519(70.2)$ & $29(74.4)$ & $490(70.0)$ \\
\hline Chronic kidney disease & $90(12.2)$ & $5(12.8)$ & $85(12.1)$ \\
\hline Rheumatological disease & $33(4.5)$ & $3(7.7)$ & $30(4.3)$ \\
\hline Dyslipidaemia & $239(32.4)$ & $10(25.6)$ & $229(32.7)$ \\
\hline Duration of hospitalisation (day), median (IQR) & $13(7-25)$ & $11(6-32)$ & $13(7-25)$ \\
\hline
\end{tabular}

*This excludes two patients with missing data.

†This presents the most frequent five reasons for admission.

¥Stroke includes ischaemic stroke, haemorrhagic stroke and subarachnoid haemorrhage.

§Ischaemic heart disease includes myocardial infarction and a history of percutaneous coronary intervention or coronary artery bypass graft surgery.

COPD, chronic obstructive pulmonary disease.

\section{DISCUSSION}

The findings of this study showed that the prevalence of PIM use at admission and discharge among elderly hospitalised patients with acute medical illnesses is high in Japan, although the proportion of patients taking any PIMs decreased significantly from admission to discharge. The use of PIMs at discharge was not associated with an increased risk of short-term unplanned readmission.
This is the first study to investigate the impact of PIMs at discharge on unplanned readmissions among hospitalised elderly patients in Japan. Our findings are consistent with those of previous studies showing no association between the use of PIMs at discharge and short-term readmissions. ${ }^{17} 192829$ Given that significant associations between healthcare outcomes, such as hospitalisation, and the use of PIM in ambulatory settings 
Table 2 Temporal changes in the prevalence of PIM use among the 739 hospitalised elderly patients from admission to discharge based on subcategories of PIMs

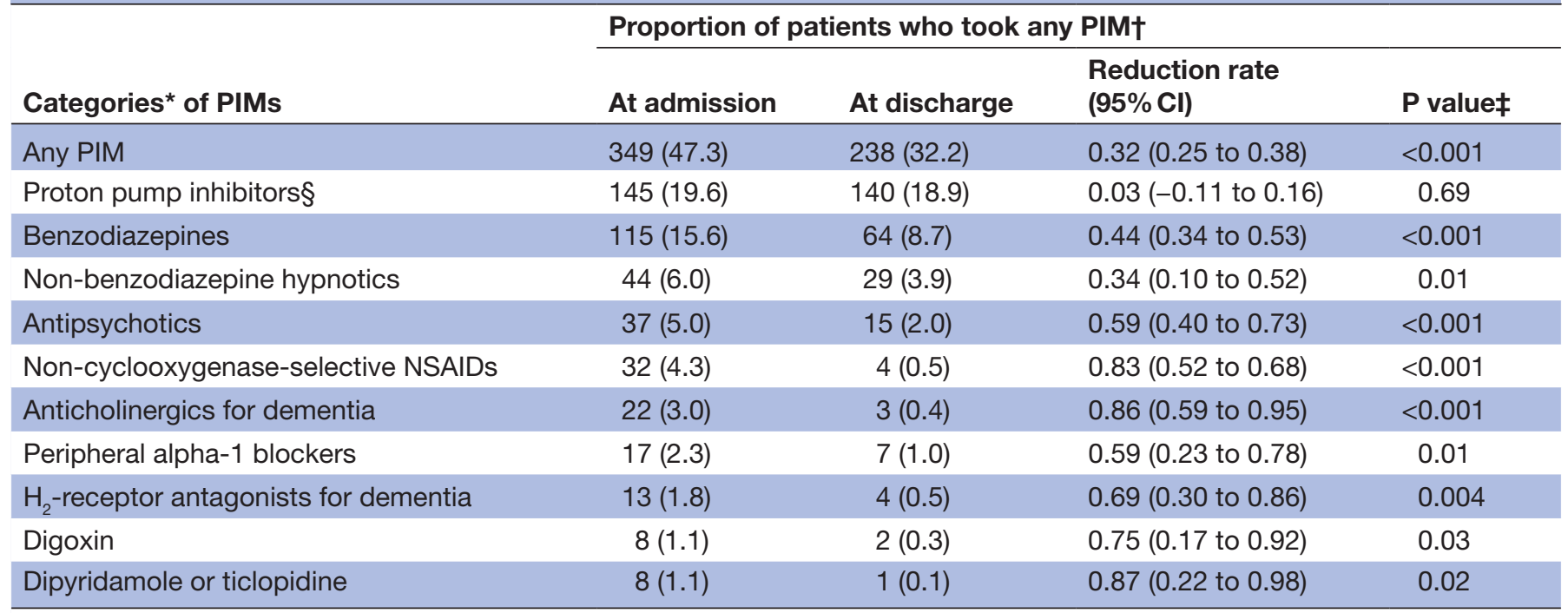

${ }^{*}$ These included subcategories of PIMs that were used in $>1 \%$ of all patients. †PIMs were defined based on the 2015 American Geriatric Society Beers Criteria.

$\ddagger$ Comparisons of the proportion of patients taking PIMs at admission and discharge were performed using the exact McNemar test. $\S$ The use of proton pump inhibitors was judged as potentially inappropriate unless there were any of the following indications: (1) active peptic ulcer disease; (2) gastro-oesophageal reflux disease; (3) eradication therapy for Helicobacter pylori; (4) pathological hypersecretory conditions; (5) use of NSAIDs or (6) use of antiplatelet therapy and at least one risk factor (history of peptic ulcer, dual antiplatelet therapy or concomitant use of anticoagulants or corticosteroids).

NSAIDs, non-steroidal anti-inflammatory drugs; PIM, potentially inappropriate medication.

and nursing care facilities have been reported in some studies ${ }^{3-6}$ the setting may affect the impact of PIMs on the healthcare outcomes among elderly patients. In fact, most randomised controlled trials ${ }^{18}{ }^{30-36}$ and prospective studies ${ }^{37} 38$ investigating the effectiveness of interventions to improve the appropriateness of medications among hospitalised elderly patients reported that the intervention can improve the appropriateness of the medications but not the frequency of unplanned readmissions. It is possible that the impact of PIMs on unplanned readmissions for elderly patients was small because other factors, such as comorbidities and premature discharge from the index hospitalisation, have largely contributed to unplanned readmissions. ${ }^{39-42}$ Furthermore, there were a few readmissions due to adverse drug reactions in this study (see the online supplementary table S3). Given that PIMs at discharge may be associated with medicationrelated readmissions rather than all-cause readmissions, ${ }^{18}$

Table 3 Univariate and multivariate analyses ${ }^{\star}$ for predictive factors of 30-day and 90-day unplanned readmissions after the index hospital discharge

\begin{tabular}{|c|c|c|c|c|}
\hline & 30-day readmission & & 90-day readmission & \\
\hline Variables & $\begin{array}{l}\text { Unadjusted OR } \\
(95 \% \mathrm{Cl})\end{array}$ & $\begin{array}{l}\text { Adjusted OR } † \\
(95 \% \mathrm{Cl})\end{array}$ & $\begin{array}{l}\text { Unadjusted OR } \\
(95 \% \mathrm{Cl})\end{array}$ & $\begin{array}{l}\text { Adjusted OR } † \\
(95 \% \mathrm{Cl})\end{array}$ \\
\hline Women & $0.43(0.21 \text { to } 0.85)^{\star}$ & $0.43(0.21 \text { to } 0.88)^{\star}$ & $0.64(0.41 \text { to } 0.98)^{*}$ & $0.63(0.40 \text { to } 0.99)^{*}$ \\
\hline Charlson Comorbidity Index $\ddagger$ & $1.38(1.18 \text { to } 1.61)^{\star \star}$ & $1.34(1.14 \text { to } 1.58)^{\star \star}$ & $1.27(1.14 \text { to } 1.42)^{\star \star}$ & $1.24(1.10 \text { to } 1.40)^{* \star}$ \\
\hline PIMs at discharge & $0.93(0.46$ to 1.87$)$ & 0.78 (0.36 to 1.66$)$ & $0.78(0.48$ to 1.24$)$ & 0.64 (0.38 to 1.07$)$ \\
\hline
\end{tabular}

The threshold for statistical significance was set at $\mathrm{p}<0.05$. Asterisks indicate a significant association between selected variables and unplanned readmissions; ${ }^{*} p<0.05,{ }^{* *} p<0.001$.

†The following variables were adjusted for: age, sex, Charlson Comorbidity Index, duration of the index hospitalisation, number of medications at discharge and PIMs at discharge.

$\ddagger$ Continuous variables were used.

§Polypharmacy was defined as five or more medications.

PIM, potentially inappropriate medication. 
the low rate of readmissions due to adverse drug reactions in the present study might have mitigated the impact of PIMs on unplanned readmissions. Nonetheless, our results should be interpreted cautiously because a 95\% CI of the OR for the association between PIM use at discharge and unplanned readmission is wide due to an unexpectedly low rate of events.

In our study, polypharmacy (defined as the use of five or more medications) at discharge was not associated with an increased risk of unplanned readmissions. This finding is not consistent with that of previous studies showing that the number of medications or polypharmacy at discharge is an independent predictive factor for unplanned readmissions, ${ }^{28}{ }^{43-47}$ although one prospective cohort study reported no association between the number of medications at discharge and unplanned readmissions. ${ }^{37}$ However, in the multivariate analysis for predictive factors for unplanned readmissions, the 95\% CI of the OR of polypharmacy was wide in this study. It is possible that a lack of power due to a low readmission rate might explain why there was no statistically significant association between polypharmacy at discharge and unplanned readmissions in this study.

The prevalence of PIM use at admission and discharge among hospitalised elderly patients in this study was high, as similar studies conducted in Japan ${ }^{11} 12$ and other countries $^{10} 13-15$ 17-19 2829 have reported. Although a reduction in the prevalence of PIM use from admission to discharge in this study was somewhat encouraging, one in three hospitalised elderly patients still received at least one PIM at discharge. Given the high prevalence of PIM use at discharge in hospitalised elderly patients, further studies are necessary to evaluate the effect of PIM use at discharge on clinical outcomes because few studies, including the present study, had a large enough sample size that was sufficient to determine the association between PIM use at discharge and clinical outcomes. ${ }^{17} 192829$

Our results are consistent with those of recent studies $^{11} 48-50$ showing that the most common PIMs among hospitalised elderly patients were PPIs and benzodiazepines. Diuretics were the second most frequently used PIM in two of those studies ${ }^{48} 49$ because PIMs were defined based on all five parts of the 2015 Beers Criteria in the two studies, unlike our study. Given the high prevalence of PIM use regarding PPIs and benzodiazepines, a strategy to focus on PPIs ${ }^{51}$ and benzodiazepines ${ }^{52} 53$ among hospitalised elderly patients is needed.

\section{Implications for clinicians}

The use of PIMs is common in an acute care setting. However, our findings and those of previous studies suggest that the use of PIMs at discharge is not associated with short-term unplanned readmissions. Nonetheless, the $95 \%$ CI of the OR for the primary outcome in this study was too wide to draw definitive conclusions. Moreover, a recent retrospective cohort study reported that hospitalisation was independently associated with potentially inappropriate prescribing in elderly patients, ${ }^{54}$ which may result in adverse events in the long term. ${ }^{3-6}$ In addition, some prospective studies have reported that interventions to improve the appropriateness of medications among hospitalised elderly patients have reduced medicationrelated hospital revisits, ${ }^{55} 56$ emergency department visits without hospitalisation, ${ }^{38}$ 30-day unplanned readmissions $^{57}$ and adverse drug reactions. ${ }^{58} 59$ Thus, given the high prevalence of PIM use, it is important to implement a strategy to improve medication appropriateness among hospitalised elderly patients.

\section{LIMITATIONS}

First, this study was limited to a single centre and to elderly patients admitted to the internal medicine ward. Therefore, these findings may not be generalised to other wards and hospitals. Second, this was not a randomised controlled design. Therefore, some confounding factors might have introduced a bias. Third, adherence to and temporal changes in medication use after discharge were not evaluated in this research. Given that transitional care after discharge often causes a discrepancy between medications at discharge and after discharge, ${ }^{39} 60$ the prescriptions at hospital discharge might not accurately reflect medications after discharge. Fourth, as-needed medications and OTC drugs were excluded in this study. Therefore, the prevalence of PIM use might have been underestimated. Fifth, all unplanned readmissions after the index hospital discharge could not be captured because we collected information only from the database of our hospital. Although the 30-day readmission rate obtained in this study was similar to that of other Japanese hospitals ${ }^{6162}$ the targeted population was somewhat different. Sixth, the 95\% CI of the ORs for the primary outcome was wide because the unplanned readmission rate was lower than we expected. Therefore, the association between any PIM use at discharge and unplanned readmissions needs to be investigated in future studies with a larger sample size or with high-risk populations for readmission. Seventh, we did not evaluate several important factors, ${ }^{40} 416364$ such as socioeconomic status and the time interval between hospital discharge and the first follow-up visit to physicians, which could affect unplanned readmissions. Eighth, we did not evaluate the preventability of unplanned readmissions. However, a recent study reported that there was no difference in the number of PIMs between hospitalised elderly patients who had experienced an avoidable readmission and those who had not. ${ }^{65}$ Ninth, an association between disease-specific $\mathrm{PIMs}^{66}$ and unplanned readmissions was not evaluated. Tenth, we adapted the definition of PIM regarding PPIs in the 2015 Beers Criteria. However, a recent study also adapted the PPI part of the 2015 Beers Criteria. ${ }^{28}$ Eleventh, we used only the first two tables of the 2015 Beers Criteria. However, previous similar studies also used only one or two parts of the 2015 Beers Criteria. ${ }^{192}$ Finally, the duration of hospital stays in Japan was longer than that in other countries. ${ }^{67}$ Therefore, our findings might not be 
applicable to countries other than Japan. However, in this study, the duration of hospitalisation was not associated with an increased risk of unplanned readmissions.

\section{CONCLUSIONS}

The proportion of patients taking any PIMs at discharge was high among hospitalised elderly patients. Although the use of PIMs at discharge was not associated with an increased risk of short-term readmission after discharge, given a lack of power of this study due to a low event rate, further studies investigating this association are needed.

Contributors JK conceived and designed this study and wrote a draft of the protocol for this study. JK, TY and MK revised the protocol. JK collected and analysed the data and wrote a draft of the main paper. JK, TY and MK discussed the results and interpretations and were involved in critical revisions of the manuscript. $\mathrm{JK}, \mathrm{TY}$ and MK read and approved the final version of the manuscript.

Funding The authors have not declared a specific grant for this research from any funding agency in the public, commercial or not-for-profit sectors.

Competing interests None declared.

Patient consent for publication Not required.

Ethics approval The protocol of this research was approved by the Medical Ethical Committee of the National Hospital Organization Tochigi Medical Center (No. 29-13). This research was conducted in accordance with the Ethical Guidelines for Epidemiological Research in Japan and the Declaration of Helsinki. The need for individual informed consent was formally waived by the Medical Ethical Committee of the National Hospital Organization Tochigi Medical Center because the de-identified data were collected from medical records without contact with the patients. However, according to the Japanese Ethical Guidelines, we displayed an opt-out statement in the waiting room and webpage of the hospital to provide the patients with information about the research and the opportunity to refuse the use of the data.

Provenance and peer review Not commissioned; externally peer reviewed.

Data availability statement All data relevant to the study are included in the article or uploaded as supplementary information.

Open access This is an open access article distributed in accordance with the Creative Commons Attribution Non Commercial (CC BY-NC 4.0) license, which permits others to distribute, remix, adapt, build upon this work non-commercially, and license their derivative works on different terms, provided the original work is properly cited, appropriate credit is given, any changes made indicated, and the use is non-commercial. See: http://creativecommons.org/licenses/by-nc/4.0/.

ORCID iD

Junpei Komagamine http://orcid.org/0000-0002-5899-4760

\section{REFERENCES}

1 O'Mahony D, O'Sullivan D, Byrne S, et al. STOPP/START criteria for potentially inappropriate prescribing in older people: version 2. Age Ageing 2015;44:213-8.

2 By the American Geriatrics Society 2015 Beers Criteria Update Expert Panel. American geriatrics Society 2015 updated beers criteria for potentially inappropriate medication use in older adults. $J$ Am Geriatr Soc 2015;63:2227-46.

3 Jano E, Aparasu RR. Healthcare outcomes associated with beers' criteria: a systematic review. Ann Pharmacother 2007;41:438-48.

4 Hyttinen V, Jyrkkä J, Valtonen H. A systematic review of the impact of potentially inappropriate medication on health care utilization and costs among older adults. Med Care 2016;54:950-64.

5 Muhlack DC, Hoppe LK, Weberpals J, et al. The association of potentially inappropriate medication at older age with cardiovascular events and overall mortality: a systematic review and meta-analysis of cohort studies. J Am Med Dir Assoc 2017;18:211-20.

6 Wang KN, Bell JS, Chen EYH, et al. Medications and prescribing patterns as factors associated with hospitalizations from long-term care facilities: a systematic review. Drugs Aging 2018;35:423-57.
7 Opondo D, Eslami S, Visscher S, et al. Inappropriateness of medication prescriptions to elderly patients in the primary care setting: a systematic review. PLoS One 2012;7:e43617.

8 Tommelein E, Mehuys E, Petrovic M, et al. Potentially inappropriate prescribing in community-dwelling older people across Europe: a systematic literature review. Eur J Clin Pharmacol 2015;71:1415-27.

9 Storms H, Marquet K, Aertgeerts B, et al. Prevalence of inappropriate medication use in residential long-term care facilities for the elderly: a systematic review. Eur J Gen Pract 2017;23:69-77.

10 Gallagher P, Lang PO, Cherubini A, et al. Prevalence of potentially inappropriate prescribing in an acutely ill population of older patients admitted to six European hospitals. Eur J Clin Pharmacol 2011;67:1175-88.

11 Komagamine J. Prevalence of potentially inappropriate medications at admission and discharge among hospitalised elderly patients with acute medical illness at a single centre in Japan: a retrospective cross-sectional study. BMJ Open 2018;8:e021152.

12 Kimura T, Ogura F, Yamamoto K, et al. Potentially inappropriate medications in elderly Japanese patients: effects of pharmacists' assessment and intervention based on screening tool of older persons' potentially inappropriate prescriptions criteria ver.2. J Clin Pharm Ther 2017;42:209-14.

13 Onatade R, Auyeung V, Scutt G, et al. Potentially inappropriate prescribing in patients on admission and discharge from an older peoples' unit of an acute UK Hospital. Drugs Aging 2013;30:729-37.

14 Laroche M-L, Charmes J-P, Nouaille Y, et al. Impact of hospitalisation in an acute medical geriatric unit on potentially inappropriate medication use. Drugs Aging 2006;23:49-59.

15 Egger SS, Bachmann A, Hubmann N, et al. Prevalence of potentially inappropriate medication use in elderly patients: comparison between general medical and geriatric wards. Drugs Aging 2006;23:823-37.

16 Juliano ACDSRS, Lucchetti ALG, Silva JTSda, et al. Inappropriate prescribing in older hospitalized adults: a comparison of medical specialties. J Am Geriatr Soc 2018;66:383-8.

17 Mansur N, Weiss A, Beloosesky Y. Is there an association between inappropriate prescription drug use and adherence in discharged elderly patients? Ann Pharmacother 2009;43:177-84.

18 Gillespie U, Alassaad A, Hammarlund-Udenaes M, et al. Effects of pharmacists' interventions on appropriateness of prescribing and evaluation of the instruments' (Mai, STOPP and starts') ability to predict Hospitalization-Analyses from a randomized controlled trial. PLoS One 2013;8:e62401.

19 Pasina L, Djade CD, Tettamanti M, et al. Prevalence of potentially inappropriate medications and risk of adverse clinical outcome in a cohort of hospitalized elderly patients: results from the REPOSI study. J Clin Pharm Ther 2014;39:511-5.

20 Parekh N, Ali K, Page A, et al. Incidence of medication-related harm in older adults after hospital discharge: a systematic review. J Am Geriatr Soc 2018;66:1812-22.

21 Parekh N, Ali K, Stevenson JM, et al. Incidence and cost of medication harm in older adults following hospital discharge: a multicentre prospective study in the UK. Br J Clin Pharmacol 2018;84:1789-97.

22 Charlson ME, Pompei P, Ales KL, et al. A new method of classifying prognostic comorbidity in longitudinal studies: development and validation. J Chronic Dis 1987;40:373-83.

23 Bergamo D, Pastorino A, Greppi F, et al. Inappropriate proton pump inhibitor prescription in elderly adults: as usual as dangerous. J Am Geriatr Soc 2015;63:2198-9.

24 Bhatt DL, Scheiman J, Abraham NS, et al. ACCF/ACG/AHA 2008 expert consensus document on reducing the gastrointestinal risks of antiplatelet therapy and NSAID use. Am J Gastroenterol 2008;103:2890-907.

25 Lanza FL, Chan FKL, Quigley EMM, et al. Guidelines for prevention of NSAID-related ulcer complications. Am J Gastroenterol 2009;104:728-38.

26 Abraham NS, Hlatky MA, Antman EM, et al. ACCF/ACG/AHA 2010 expert consensus document on the concomitant use of proton pump inhibitors and thienopyridines: a focused update of the ACCF/ ACG/AHA 2008 expert consensus document on reducing the gastrointestinal risks of antiplatelet therapy and NSAID use. Am J Gastroenterol 2010;105:2533-49.

27 Gnjidic D, Hilmer SN, Blyth FM, et al. Polypharmacy cutoff and outcomes: five or more medicines were used to identify communitydwelling older men at risk of different adverse outcomes. J Clin Epidemiol 2012;65:989-95.

28 Fabbietti P, Di Stefano G, Moresi R, et al. Impact of potentially inappropriate medications and polypharmacy on 3-month readmission among older patients discharged from acute care Hospital: a prospective study. Aging Clin Exp Res 2018;30:977-84. 
29 Counter D, Millar JWT, McLay JS. Hospital readmissions, mortality and potentially inappropriate prescribing: a retrospective study of older adults discharged from hospital. Br J Clin Pharmacol 2018;84:1757-63.

30 Spinewine A, Swine C, Dhillon S, et al. Effect of a collaborative approach on the quality of prescribing for geriatric inpatients: a randomized, controlled trial. J Am Geriatr Soc 2007;55:658-65.

31 Gallagher PF, O'Connor MN, O'Mahony D. Prevention of potentially inappropriate prescribing for elderly patients: a randomized controlled trial using STOPP/START criteria. Clin Pharmacol Ther 2011;89:845-54.

32 Cossette B, Éthier J-F, Joly-Mischlich T, et al. Reduction in targeted potentially inappropriate medication use in elderly inpatients: a pragmatic randomized controlled trial. Eur J Clin Pharmacol 2017;73:1237-45.

33 Thomas R, Huntley AL, Mann M, et al. Pharmacist-Led interventions to reduce unplanned admissions for older people: a systematic review and meta-analysis of randomised controlled trials. Age Ageing 2014;43:174-87.

34 Walsh KA, O'Riordan D, Kearney PM, et al. Improving the appropriateness of prescribing in older patients: a systematic review and meta-analysis of pharmacists' interventions in secondary care. Age Ageing 2016;45:201-9.

35 Renaudin P, Boyer L, Esteve M-A, et al. Do pharmacist-led medication reviews in hospitals help reduce hospital readmissions? A systematic review and meta-analysis. Br J Clin Pharmacol 2016;82:1660-73.

36 Rankin A, Cadogan CA, Patterson SM, et al. Interventions to improve the appropriate use of polypharmacy for older people. Cochrane Database Syst Rev 2018;9.

37 Urfer M, Elzi L, Dell-Kuster S, et al. Intervention to improve appropriate prescribing and reduce polypharmacy in elderly patients admitted to an internal medicine unit. PLoS One 2016;11:e0166359.

38 Van der Linden L, Decoutere L, Walgraeve K, et al. Combined use of the rationalization of home medication by an adjusted STOPP in older patients (RASP) list and a pharmacist-led medication review in very old inpatients: impact on quality of prescribing and clinical outcome. Drugs Aging 2017;34:123-33.

39 Auerbach AD, Kripalani S, Vasilevskis EE, et al. Preventability and causes of readmissions in a national cohort of general medicine patients. JAMA Intern Med 2016;176:484-94.

40 Kansagara D, Englander $\mathrm{H}$, Salanitro $\mathrm{A}$, et al. Risk prediction models for hospital readmission: a systematic review. JAMA 2011;306:1688-98

41 Zhou H, Della PR, Roberts P, et al. Utility of models to predict 28-day or 30-day unplanned hospital readmissions: an updated systematic review. BMJ Open 2016;6:e011060.

42 Moore C, Wisnivesky J, Williams S, et al. Medical errors related to discontinuity of care from an inpatient to an outpatient setting. J Gen Intern Med 2003;18:646-51.

43 Morandi A, Bellelli G, Vasilevskis EE, et al. Predictors of rehospitalization among elderly patients admitted to a rehabilitation Hospital: the role of polypharmacy, functional status, and length of stay. J Am Med Dir Assoc 2013;14:761-7.

$44 \mathrm{Pal} \mathrm{A}$, Babbott S, Wilkinson ST. Can the targeted use of a discharge pharmacist significantly decrease 30-day readmissions? Hosp Pharm 2013;48:380-8.

45 Aljishi M, Parekh K. Risk factors for general medicine readmissions and association with mortality. N Z Med J 2014;127:42-50.

46 Picker D, Heard K, Bailey TC, et al. The number of discharge medications predicts thirty-day Hospital readmission: a cohort study. BMC Health Serv Res 2015;15:282.

47 Raval AD, Zhou S, Wei W, et al. 30-Day readmission among elderly Medicare beneficiaries with type 2 diabetes. Popul Health Manag 2015;18:256-64.

48 Zhang X, Zhou S, Pan K, et al. Potentially inappropriate medications in hospitalized older patients: a cross-sectional study using the beers 2015 criteria versus the 2012 criteria. Clin Interv Aging 2017:12:1697-703.

49 Ma Z, Zhang C, Cui X, et al. Comparison of three criteria for potentially inappropriate medications in Chinese older adults. Clin Interv Aging 2019;14:65-72.

50 Bo M, Gibello M, Brunetti E, et al. Prevalence and predictors of inappropriate prescribing according to the Screening Tool of Older People's Prescriptions and Screening Tool to Alert to Right Treatment version 2 criteria in older patients discharged from geriatric and internal medicine wards: A prospective observational multicenter study. Geriatr Gerontol Int 2019;19:5-11.

51 Wilsdon TD, Hendrix I, Thynne TRJ, et al. Effectiveness of interventions to deprescribe inappropriate proton pump inhibitors in older adults. Drugs Aging 2017;34:265-87.

52 Reeve $\mathrm{E}$, Ong M, Wu A, et al. A systematic review of interventions to deprescribe benzodiazepines and other hypnotics among older people. Eur J Clin Pharmacol 2017;73:927-35.

53 Wilson MG, Lee TC, Hass A, et al. Empowering hospitalized older adults to deprescribe sedative hypnotics: a pilot study. J Am Geriatr Soc 2018;66:1186-9.

54 Pérez T, Moriarty F, Wallace E, et al. Prevalence of potentially inappropriate prescribing in older people in primary care and its association with hospital admission: longitudinal study. BMJ 2018;86.

55 Gillespie U, Alassaad A, Henrohn D, et al. A comprehensive pharmacist intervention to reduce morbidity in patients 80 years or older: a randomized controlled trial. Arch Intern Med 2009;169:894-900.

56 Hellström LM, Bondesson A, Höglund P, et al. Impact of the Lund integrated medicines management (LIMM) model on medication appropriateness and drug-related Hospital revisits. Eur J Clin Pharmacol 2011:67:741-52.

57 Chiu P, Lee A, See T, et al. Outcomes of a pharmacist-led medication review programme for hospitalised elderly patients. Hong Kong Med $J$ 2018;24:98-106.

58 Wehling $\mathrm{M}$, Burkhardt $\mathrm{H}$, Kuhn-Thiel A, et al. VALFORTA: a randomised trial to validate the FORTA (fit fOR the aged) classification. Age Ageing 2016;45:262-7.

59 O'Connor MN, O'Sullivan D, Gallagher PF, et al. Prevention of hospital-acquired adverse drug reactions in older people using screening tool of older persons' prescriptions and screening tool to alert to right treatment criteria: a cluster randomized controlled trial. $J$ Am Geriatr Soc 2016;64:1558-66.

60 Kripalani S, Jackson AT, Schnipper JL, et al. Promoting effective transitions of care at hospital discharge: a review of key issues for hospitalists. J. Hosp. Med. 2007;2:314-23.

61 Otsubo T, Imanaka Y. [Readmission rate for health care delivery system assessment]. Nihon Eiseigaku Zasshi 2012;67:62-6.

62 Aizawa H, Imai S, Fushimi K. Factors associated with 30-day readmission of patients with heart failure from a Japanese administrative database. BMC Cardiovasc Disord 2015;15:134.

63 Hernandez AF, Greiner MA, Fonarow GC, et al. Relationship between early physician follow-up and 30-day readmission among Medicare beneficiaries hospitalized for heart failure. JAMA 2010;303:1716-22.

64 Hansen LO, Young RS, Hinami K, et al. Interventions to reduce 30-day rehospitalization: a systematic review. Ann Intern Med 2011;155:520-8

65 Blanc A-L, Spasojevic S, Leszek A, et al. A comparison of two tools to screen potentially inappropriate medication in internal medicine patients. J Clin Pharm Ther 2018;43:232-9.

66 Bermingham M, Ryder M, Travers B, et al. The St Vincent's potentially inappropriate medicines study: development of a diseasespecific consensus list and its evaluation in ambulatory heart failure care. Eur J Heart Fail 2014;16:915-22.

67 Tiessen J, Kambara H, Sakai T, et al. What causes international variations in length of stay: a comparative analysis for two inpatient conditions in Japanese and Canadian hospitals. Health Serv Manage Res 2013;26:86-94. 